\title{
Spore morphology of Taenitis, Syngramma, and Austrogramme species (Pteridoideae, Pteridaceae) from South-Eastern Asia and Oceania. II
}

\author{
A. V. Vaganov ${ }^{1,3 *}$, I. I. Gureyeva ${ }^{2,5}$, A. I. Shmakov ${ }^{1,6}$, A. A. Kuznetsov ${ }^{2,7}$ R. S. Romanets ${ }^{2,8}$ \\ ${ }^{1}$ South Siberian Botanical Garden, Altai State University, Lenina pr., 61, Barnaul, 656049, Russia; \\ ${ }^{2}$ Tomsk State University, Lenina pr., 36, Tomsk, 634050,Russia.E-mail: gureyeva@yandex.ru \\ ${ }^{3}$ Sakhalin Branch of the Botanical Garden Institute of the Far Eastern Branch of the Russian Academy \\ of Sciences, Gorkogo st., 25, Yuzhno-Sakhalinsk, 693023, Russia. \\ ${ }^{4}$ ORCID iD: https://orcid.org/0000-0002-7584-5150; 5 ORCID iD: https://orcid.org/0000-0002-5397-6133; \\ ${ }^{6}$ ORCID iD: https://orcid.org/0000-0002-1052-4575; ${ }^{7}$ ORCID iD: https://orcid.org/0000-0002-5553-2958 \\ ${ }^{8}$ ORCID iD: https://orcid.org/0000-0003-3934-6079 \\ *Corresponding author. E-mail: vaganov_vav@mail.ru
}

Keywords: Austrogramme, Pteridaceae, Pteridoideae, scanning electronic microscopy (SEM), spore morphology, Syngramma, Taenitis.

Summary. This paper continues consideration of the spores of three paleotropical fern genera - Taenitis, Syngramma, and Austrogramme (Pteridoideae, Pteridaceae) from South-Eastern Asia and Oceania. At the second stage, we carried out a comparative scanning electron microscopy study of spores of three species of Austrogramme, four species of Syngramma, and six species of Taenitis and added information about previously studied spores of seven species of these genera. Spores of all examined species are trilete, tetrahedral or tetrahedral-globose with convex to hemispherical distal side and plane, convex or conical proximal side. The spores of Austrogramme species are the smallest, simplest in ornamentation and similar to each other. Sculpture of the proximal and distal sides are microverrucate, the surface of the spores is covered by granular deposits. Spores of most Syngramma species are very similar to spores of Austrogramme species in shape and surface sculpture: their distal and proximal surfaces are microverrucate, whereas the spores of S. borneensis and S. cartilagidens have the low-tuberculate sculpture. Spores of Taenitis species are very different from the spores of Austrogramme and Syngramma. Seven of nine studied species have spores with well-expressed cingulum (T. blechnoides, T. cordata, T. diversifolia, T. interrupta, T. luzonica, T. obtusa, and T. requiniana), three species (T. cordata, T. hookeri, and T. pinnata) have spores with prominent laesural ridges. The spores have well-expressed ornamentation - tuberculate, baculate, rugate, tuberculate-rugate. The most conspicuous character of the ornamentation of spore surfaces is the presence of rodlets associated with sculpture elements. The densest rodlets are characteristic of Taenitis diversifolia, T. luzonica, T. obtusa, and T. requiniana. Spore size (equatorial diameter) ranges on average between $22 \mu \mathrm{m}$ and $37 \mu \mathrm{m}$ in Austrogramme, between $27 \mu \mathrm{m}$ and $41 \mu \mathrm{m}$ in Syngramma, and between 26 and $51 \mu \mathrm{m}$ in Taenitis species.

\section{Морфология спор Taenitis, Syngramma и Austrogramme (Pteridoideae, Pteridaceae) из Юго-Восточной Азии и Океании. II}

\author{
А. В. Ваганов ${ }^{1,3}$, И. И. Гуреева ${ }^{2}$ А. И. Шмаков ${ }^{1}$, А. А. Кузнецов ${ }^{2}$ Р. С. Романец ${ }^{2}$ \\ ${ }^{1}$ Южно-Сибирский ботанический сад, Алтайский государственный университет, просп. Ленина, 61, \\ г. Барнаул, 656049, Россия \\ ${ }^{2}$ Томский государственный университет, просп. Ленина, 36, г. Томск, 634050, Россия
}


${ }^{3}$ Сахалинский филиал Ботанического сада-института ДВО РАН, ул. Горького, 21, г. Южно-Сахалинск, 693023, Россия

Ключевые слова: морфология спор, сканирующая электронная микроскопия (СЭМ), Austrogramme, Pteridaceae, Pteridoideae, Syngramma, Taenitis.

\begin{abstract}
Аннотация. Статья продолжает публикацию данных о морфологии спор трех палеотропических родов папоротников - Taenitis, Syngramma и Austrogramme (Pteridoideae, Pteridaceae) из Юго-Восточной Азии и Океании. На втором этапе методом сканирующей электронной микроскопии (СЭМ) проведено сравнительное исследование спор 3 видов Austrogramme, 4 видов Syngramma и 6 видов Taenitis, добавлены сведения о спорах 7 видов этих родов, изученных ранее. Споры всех изученных видов трехлучевые, тетраэдрические до почти шаровидных с выпуклой или полусферической дистальной и уплощенной, выпуклой или конической проксимальной сторонами. Споры видов Austrogramme самые мелкие, с наиболее простой скульптурой, сходны по морфологии. Скульптура спор микробородавчатая, на поверхности имеются гранулярные отложения. Споры большинства видов Syngramma имеют микробородавчатую скульптуру, по форме и скульптуре поверхности они сходны со спорами видов Austrogramme; у S. borneensis и S. cartilagidens споры бугорчатые, бугры низкие. Споры видов Taenitis значительно отличаются от спор видов Austrogramme и Syngramma. Семь из девяти исследованных видов (Taenitis blechnoides, T. cordata, T. diversifolia, T. interrupta, T. luzonica, T. obtusa и T. requiniana) имеют споры с отчетливо выраженным цингулюмом (экваториальная складка), для спор трех видов T. cordata, T. hookeri и T. pinnata - характерны выраженные комиссуральные складки. Споры имеют хорошо выраженную скульптуру - бугорчатую, удлиненно-бугорчатую, складчатую или бугорчато-складчатую. Наиболее заметным признаком орнаментации спор видов Taenitis являются стерженьки, густо покрывающие скульптурные элементы спор. Наиболее густо покрыта стерженьками поверхность спор у Taenitis diversifolia, T. luzonica, T. obtusa и T. requiniana. Размеры спор (экваториальный диаметр) варьируют в среднем в пределах 22-37 мкм у Austrogramme, 27-41 мкм у Syngramma и 26-51 мкм у Taenitis.
\end{abstract}

This paper continues consideration of spore morphology of three paleotropical fern genera Taenitis Willd. ex Schkuhr, Syngramma J. Sm., and Austrogramme E. Fourn. R. E. Holttum (1975) was the first, who studied the relationship between Taenitis and Syngramma and considered them to the Gymnogrammeoid ferns. Different authors considered these three fern genera as belonging to the separate family Taenitidaceae (C. Presl) Pic. Serm. or classified them as members of Taenitidoideae R. M. Tryon subfamily of the large and diverse family Pteridaceae E. D. M. Kirchn (Pichi Sermolli, 1977; Tryon, 1986; Tryon et al., 1990; SánchezBaracaldo, 2004; Smith et al., 2006). E. Schuettpelz et al. (2007) considered them as the members of the pteridoid ferns within the Pteridaceae. M. J. M. Christenhusz et al. (2011) and L. Zhang et al. $(2015,2017)$ classify these three genera to subfamily Pteridoideae C. Chr. ex Crabbe, Jermy et Mickel. The last to date PPG-system (PPG1, 2016) classifies these genera to suborder Pteridineae J. Prado et. Schuettp., family Pteridaceae and subfamily Pteridoideae Link sensu Zhang et al. (2015). This system recognizes all three genera sensu Tryon et al. (1990); each genus is considered as monophyletic (Taenitis and Austrogramme) (Cochran et al., 2014) or assumed to be monophyletic (Syngramma). Within Austrogramme, this system recognizes about six species, within Taenitis and Syngramma - about
15 species in each. M. Kato (1988) found the genus Taenitis consists of 17 species.

The representatives of Austrogramme occur in New Caledonia, New Guinea, the Moluccas, and the New Hebrides. Species of Syngramma inhabit Malaya to the Philippine Islands, New Guinea, and east to Fiji and the Caroline Islands (Palau), seven species occur in Borneo. The Taenitis species are distributed from southern India and Ceylon to Hainan, through Malesia to northern Queensland and Fiji, nine of them occur in Borneo (POWO. URL: http://powo.science.kew.org/).

The molecular-phylogenetic studies of the last decade based on six plastid regions (atpA, atpB, $r b c L$, rps 4 genes, rps4-trnS and trnL-F intergenic spacers, and trnL intron) confirmed the close relationship among three paleotropic genera Austrogramme, Syngramma, and Taenitis. In all published phylograms, these three genera form monophyletic clade, where Taenitis is sister to Austrogramme and Syngramma (Cochran et al., 2014; Zang et al., 2015, 2017).

Spore morphology of species of the large fern family Pteridaceae has been discussed in the numerous works (Hennipman, 1975; Tryon, Lugardon, 1991; Palacios-Rios et al., 2016; Chao, Huang, 2018; Irfan et al., 2021) including ours (Kuznetsov et al., 2014; Vaganov, 2016; Vaganov et al., 2017a-d, 2018a). Most authors emphasize 
the diagnostic value of spore ornamentation at the specific or section levels. The congruence of morphological characteristics of spores with the result of molecular-phylogenetic analyzes has been shown for different fern genera on various examples (Gureyeva, Kuznetsov, 2015; Vaganov et al., 2017a-c). Data of spore morphology of some species of Austrogramme, Syngramma, and Taenitis obtained using scanning electron microscopy were published in 1991 by A. Tryon and B. Lugardon (11 species in sum). Based on the spore structure of 16 species of these three genera, E. Hennipman (1975) considered the study of the spores to be promising for comprehension of the relationships within Pteridaceae. We also have earlier given the spore characteristics of seven species of these genera (Vaganov et al., 2018b).

The aim of this work is a comparative morphological study of the spores of Taenitis, Syngramma, and Austrogramme using scanning electron microscopy (SEM) and evaluation, whether the spore characteristics have the value for phylogeny of these genera.

\section{Materials and methods}

Mature spores for SEM examination were obtained from herbarium specimens of 11 species stored in P (Paris vascular plant Herbarium Muséum national d'Histoire naturelle, MNHN, Paris, France): Taenitis cordata (Gaudich.) Holttum, T. diversifolia Holttum, T. luzonica (Alderw.) Holttum., T. obtusa Hook., T. requiniana (Gaudich.) Copel., Syngramma grandis (Copel.) C. Chr., S. spathulata (C. Chr.) Holttum, S. wallichii (Hook.) Bedd., Austrogramme decipiens (Mett.) Hennipman, A. francii (Rosenst.) Hennipman, and A. marginata (Mett.) E. Fourn, and two species stored in PE (Herbarium of Institute of Botany, Chinese Academy of Sciences, Beijing, China): Taenitis interrupta Hook. et Grev. and Syngramma cartilagidens (Bak.) Diels. Furthermore, we used the data on spore morphology obtained by us earlier (Vaganov et al., 2018) for Austrogramme boerlageana (Alderw.) Hennipman, Syngramma alismifolia (Presl) J. Sm., S. lobbiana (Hook.) J. Sm., S. quinata (Hook.) Carruth., Taenitis blechnoides (Willd.) Sw., T. hookeri (C. Chr.) Holttum, T. pinnata (J. Sm.) Holttum. As a result, the spore morphology of four species of Austrogramme, eight species of Syngramma, and nine species of Taenitis were studied.

The spelling of the names of taxa and authors is given according to "The International Plant Name Index" (IPNI, http://www.ipni.org).
The SEM studies were conducted in the Institute for Water and Environmental Problems (Barnaul, Russia) using a "Hitachi S 3400 N" electronic scanning microscope and in Tomsk State University (Tomsk, Russia) with the scanning electronic microscope "Mini-SEM SNE-4500M". Spores were applied on double-sided carbon adhesive tape, fastened on the object table with a diameter of 10 $\mathrm{mm}$. The spore surface was coated in the sputtercoaters "Emitech SC 7620/QT S" (with the goldpalladium mixture) and "Quorum Q150R S" (with gold). The spore surface was scanned in a high vacuum at a voltage of $20 \mathrm{kV}$ through $2000 \times$ and $15000 \times$ of magnification.

Equatorial diameter (as seen in the polar position of spore), polar axis (as seen in the equatorial position of spore), length and width of laesura arms (on the proximal position of spore), the width of cingulum (as seen in the polar position of spore) were measured for 10-20 spores of each species. All measurements were made on SEM-micrographs of spores using the computer program "Image J".

The shape of the distal and proximal sides was described using terms of B. K. Nayar, S. Devi (1966). The following terms were used to denote structural elements of the spores (combination of terms used by Nayar, Devi, 1966; Tryon, Lugardon, 1991; Lellinger, 2002; Palacios-Rios et al., 2016; Chao, Huang, 2018):

- cingulum (equatorial flange, equatorial ridge) a solid continuous, thickened portion of the exospore at the equator of the spore separating the proximal and distal sides of spore;

- laesura arms - three thickened or unthickened scars on the surface of spore;

- laesural ridge (commissural ridge, commissural flange, ridge-like torus) - conspicuous ridges flanked the laesura arms and parallel of them;

sculptural elements:

- verrucae - small elements, irregular in form, obtuse to round on apex; surface verrucate (covered with verrucae) or microverrucate (covered with very small verrucae);

- tubercle - knob-like elements regular in form; surface tuberculate (covered with tubercles);

- baculae - elements $1.5 \times$ longer than wide, cylindrical, with obtuse to round apex; surface baculate (covered with baculae);

- rugae - wide rounded folds; surface rugate (covered with rugae);

- rugulae - small rugae (wrinkles); surface rugulate (covered with rugulae) or microrugulate (covered with very small rugulae); 
ultrastructures on the outer spore layer:

- rodlets - thin, short, filiform structure, situated on the outer spore layer;

- granulate deposits - small excretion on the outer spore layer.

\section{Results and discussion}

Spores of all examined species of Austrogramme, Syngramma, and Taenitis are trilete, tetrahedral or tetrahedral-globose. In equatorial position, the distal side is convex to hemispherical, proximal side is flat, convex or conical. In polar position, spores are triangular, roundish-triangular or nearly roundish with straight or concave sides and rounded corners. Spores with cingulum or without it. Ornamentation of both sides of the spore may be verrucate, tuberculate, baculate, or rugate, spore surface covered by rodlets and granulate deposits. In spores of some species, the laesura arms are flanked by laesural ridges that can be well-defined entire or interrupted. The main morphological and biometrical characteristics of spores are shown in tables 1 and 2 .

The spores of the Austrogramme species are the smallest, simplest in ornamentation and similar to each other. Spores are tetrahedral-globose in equatorial view, often with depressions on the proximal side between laesura arms. In polar position, spores are triangular-roundish or nearly roundish with straight, concave or convex sides. The proximal and distal sides are microverrucate, with verrucae $0.2-0.4 \mu \mathrm{m}$ in diam. in A. boerlageana, or verrucate, with verrucae $0.4-1.2 \mu \mathrm{m}$ in diam. in A. decipiens, $A$. francii, and $A$. marginata. The surface of the spores of three species is covered by granular deposits, dense in $A$. boerlageana, scattered in $A$. francii and rare in $A$. marginata. The spore surface of $A$. decipiens is covered by rodlets rather than granular deposits. Laesura arms are straight, $0.6-1.1 \mu \mathrm{m}$ in length. In spores of $A$. marginata, laesura arms immersed in the narrow laesural ridges that are reached to the spore corners and covered by scattered verrucae (Fig. 1, Table 1). The equatorial diameter ranges between $22.5 \mu \mathrm{m}$ and $36.7 \mu \mathrm{m}$ and the polar axis - between $14.8 \mu \mathrm{m}$ and $20 \mu \mathrm{m}$. The smallest spores are characteristic of $A$. francii and A. marginata (Table 2).

The spores of most Syngramma species are very similar to spores of the Austrogramme species in shape and surface sculpture. In equatorial view, spores of Syngramma alismifolia, S. borneensis, $S$. cartilagidens, and $S$. wallichii are tetrahedralglobose with depressions between laesura arms on the proximal side. In polar position, spores are triangular, roundish-triangular or nearly roundish with straight, slightly concave or convex sides and wide-rounded corners. Distal and proximal surfaces are microverrucate in $S$. quinata, S. lobbiana,
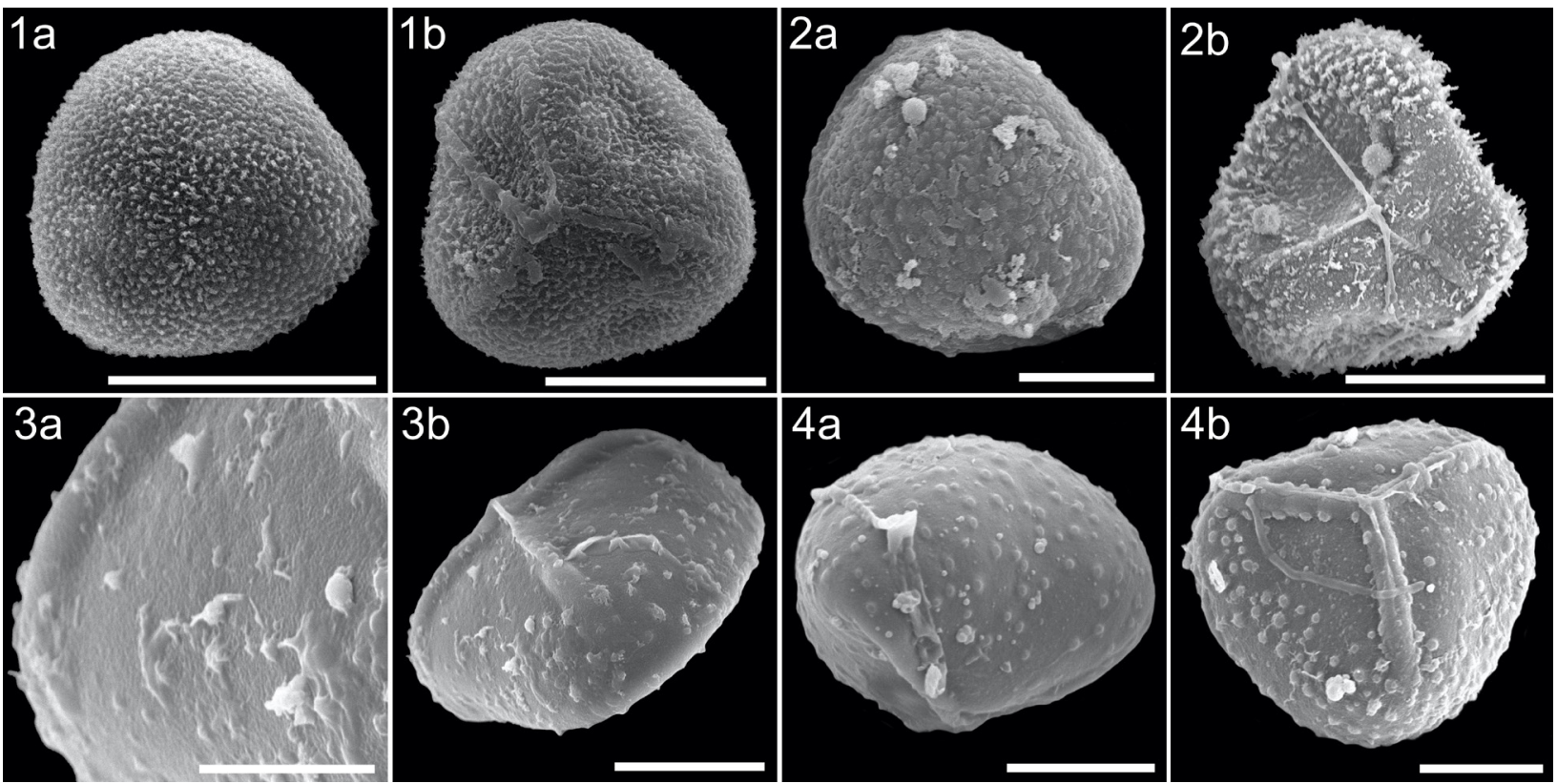

Fig. 1. SEM-micrographs of the spores of Austrogramme E. Fourn.: 1 - Austrogramme boerlageana (Alderw.) Hennipman: $\mathrm{a}$ - distal side; $\mathrm{b}$ - proximal side; 2 - Austrogramme decipiens (Mett.) Hennipman: $\mathrm{a}$ - distal side; $\mathrm{b}$ - proximal side; 3 - Austrogramme francii (Rosenst.) Hennipman: a - fragment of equatorial-proximal side of spore and laesura arms, $\mathrm{b}$ - spore in equatorial-proximal position; 4 - Austrogramme marginata (Mett.) E. Fourn.: a - spore in equatorial-distal position, $\mathrm{b}$ - proximal side. $1 \mathrm{a}, 1 \mathrm{~b}, 2 \mathrm{~b}-20 \mu \mathrm{m} ; 2 \mathrm{a}, 3 \mathrm{~b}, 4 \mathrm{a}, 4 \mathrm{~b}-10 \mu \mathrm{m} ; 3 \mathrm{a}-5 \mu \mathrm{m}$. 
$\frac{\overline{0}}{\frac{0}{0}}$

\begin{tabular}{|c|c|c|c|c|c|c|c|c|c|c|}
\hline 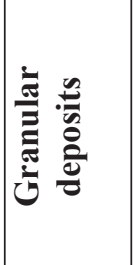 & 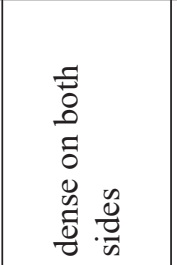 & & 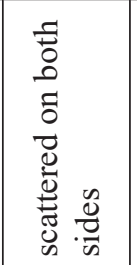 & 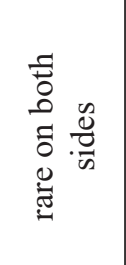 & 1 & 1 & 1 & 1 & 1 & \\
\hline 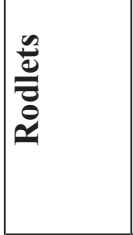 & I & 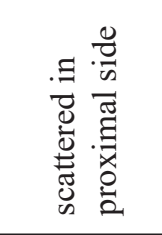 & 1 & 1 & 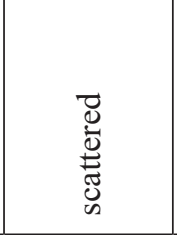 & 1 & 1 & 1 & 1 & 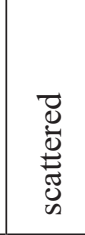 \\
\hline 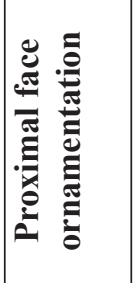 & 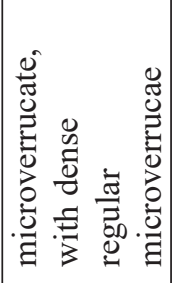 & 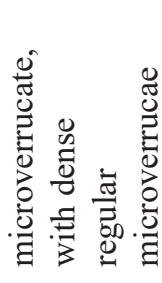 & 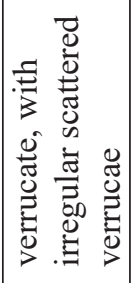 & 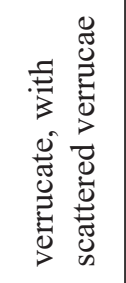 & 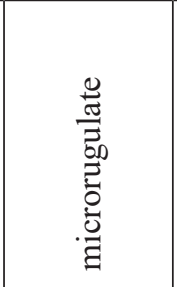 & 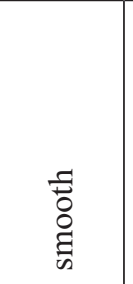 & 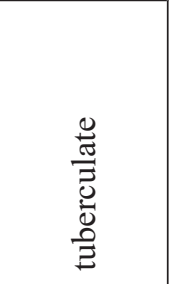 & 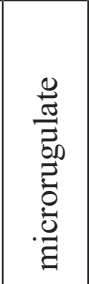 & 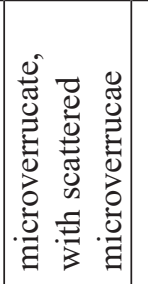 & 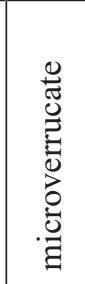 \\
\hline 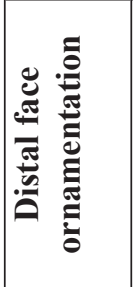 & 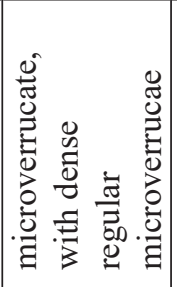 & 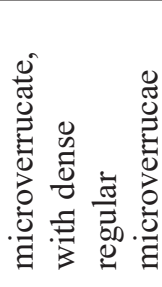 & 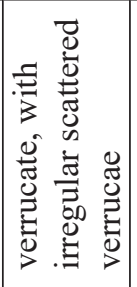 & 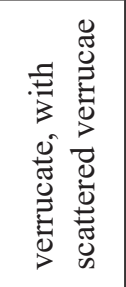 & 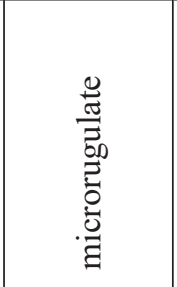 & 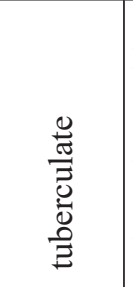 & 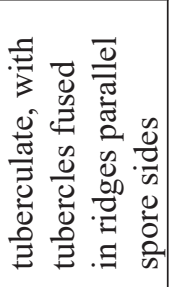 & 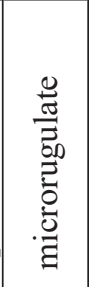 & 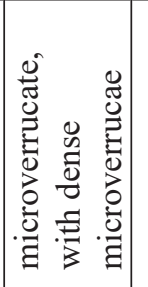 & 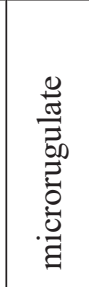 \\
\hline 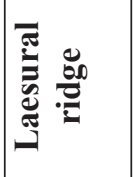 & 1 & 1 & 1 & 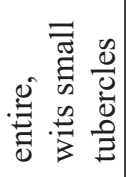 & 1 & 1 & 1 & I & 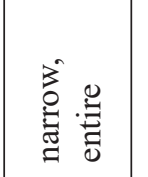 & \\
\hline $\begin{array}{l}E \\
\vdots \\
\vdots \\
0 \\
0 \\
0\end{array}$ & 1 & 1 & I & 1 & 1 & 1 & 1 & 1 & 1 & \\
\hline 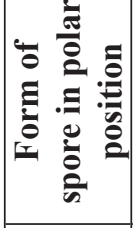 & 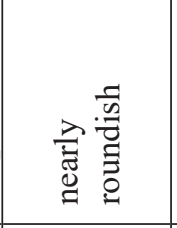 & 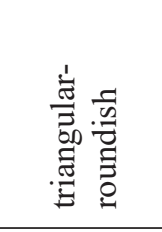 & 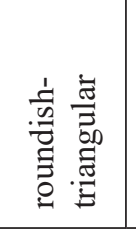 & 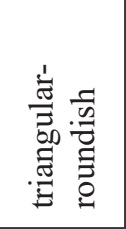 & 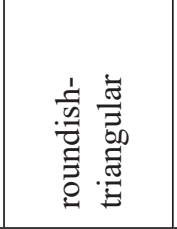 & 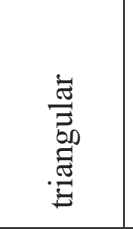 & 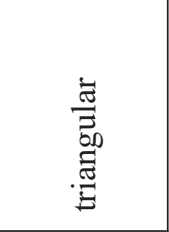 & 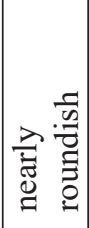 & 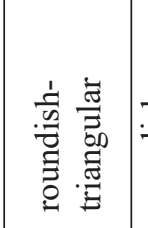 & 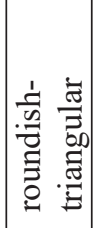 \\
\hline 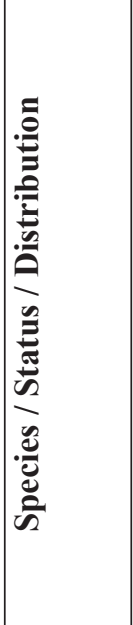 & 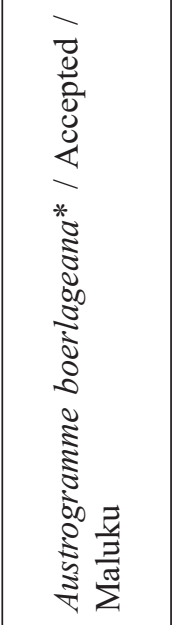 & 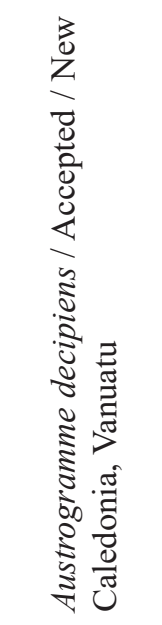 & 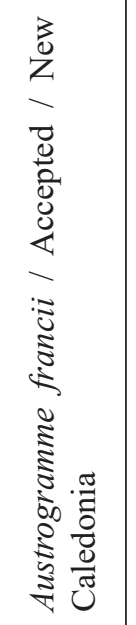 & 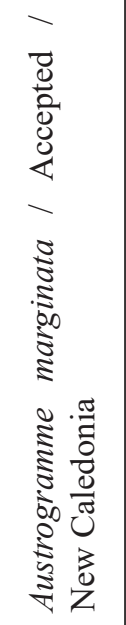 & 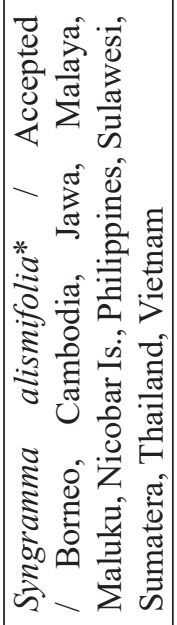 & 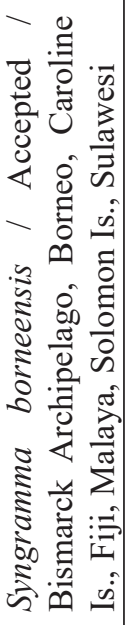 & 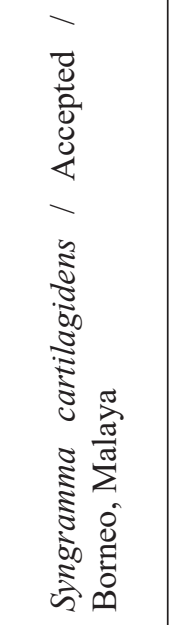 & 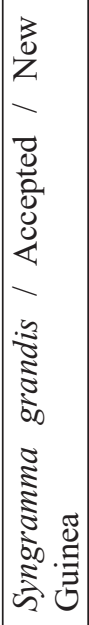 & 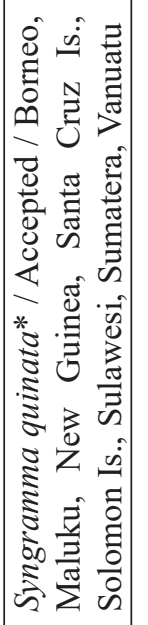 & 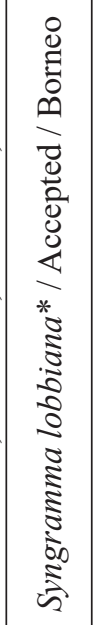 \\
\hline
\end{tabular}




\begin{tabular}{|c|c|c|c|c|c|c|}
\hline 章 & 1 & 1 & 1 & 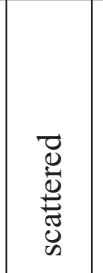 & 1 & 1 \\
\hline 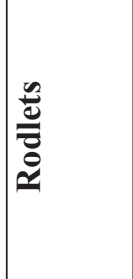 & 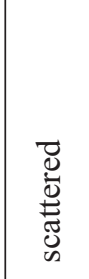 & 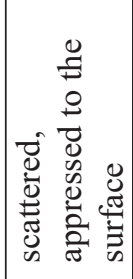 & 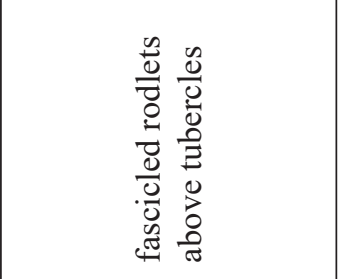 & . & 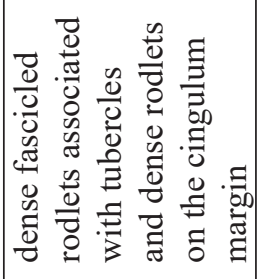 & 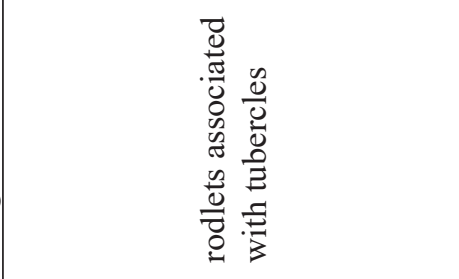 \\
\hline 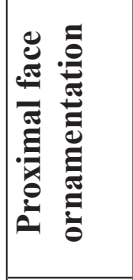 & 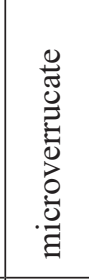 & 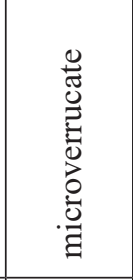 & 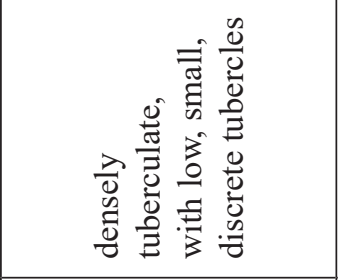 & 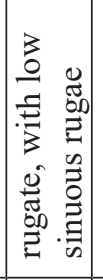 & 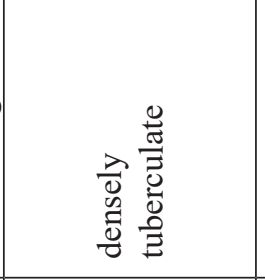 & 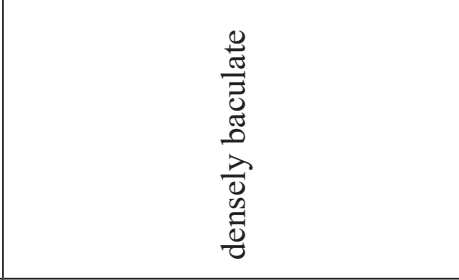 \\
\hline 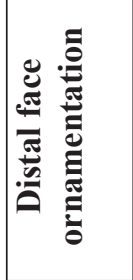 & 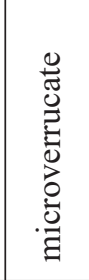 & 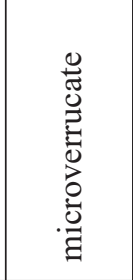 & 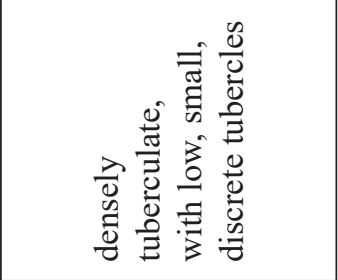 & 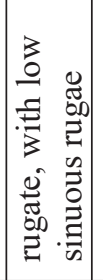 & 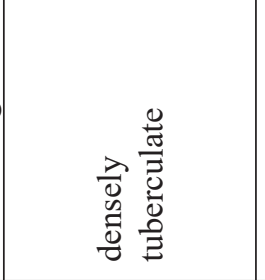 & 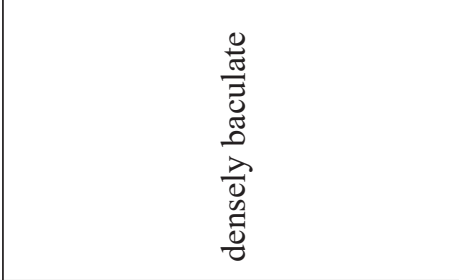 \\
\hline 莡 & 1 & I & I & 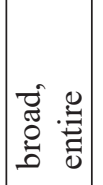 & I & 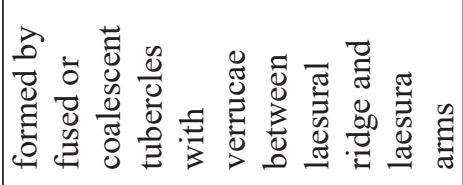 \\
\hline Uू & 1 & 1 & 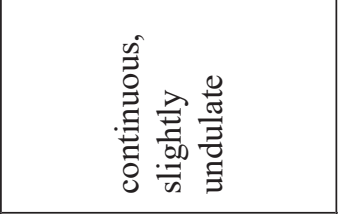 & 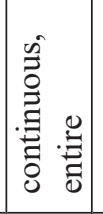 & 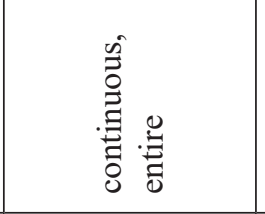 & 1 \\
\hline 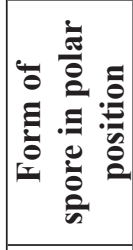 & 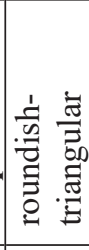 & 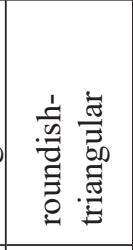 & 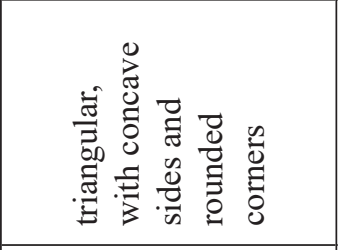 & 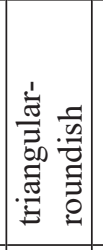 & 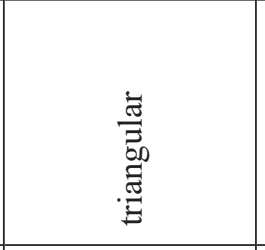 & 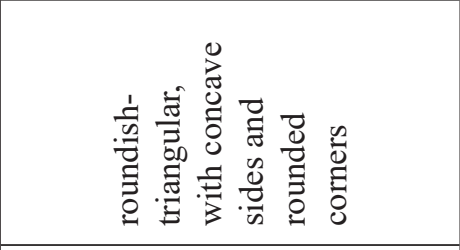 \\
\hline 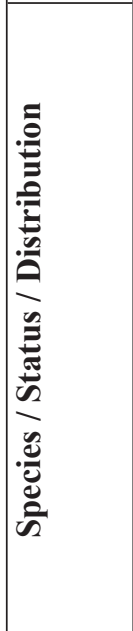 & 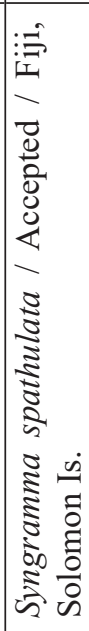 & 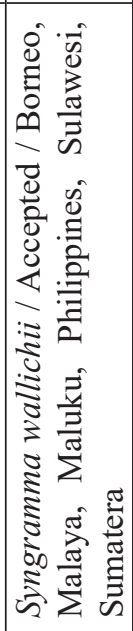 & 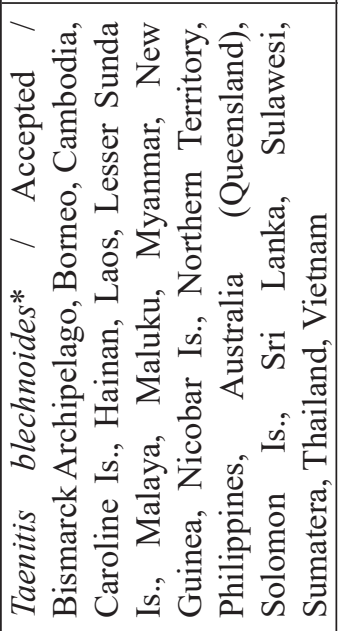 & 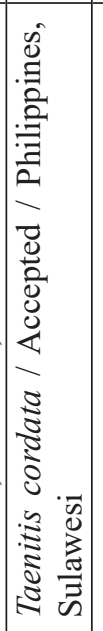 & 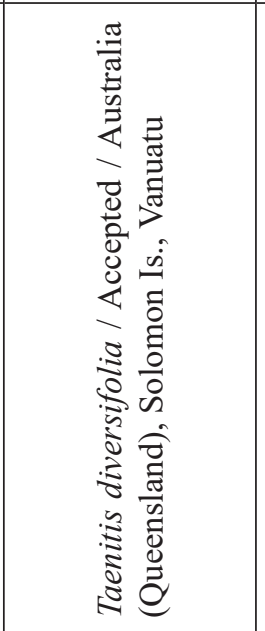 & 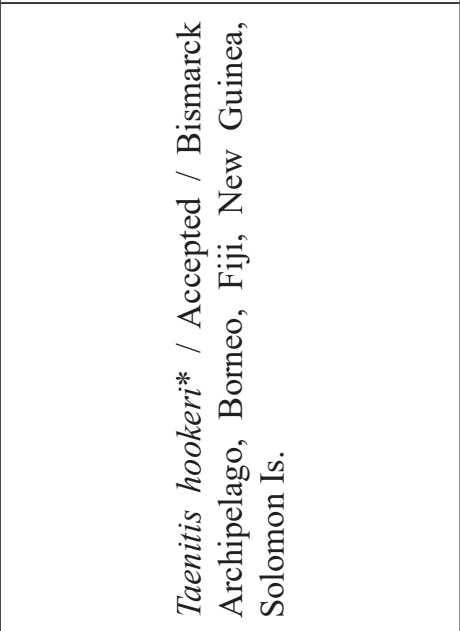 \\
\hline
\end{tabular}


बृ
ब्व
$\frac{0}{0}$

\begin{tabular}{|c|c|c|c|c|c|}
\hline 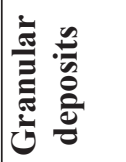 & 1 & 1 & 1 & & 1 \\
\hline 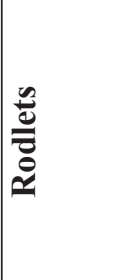 & 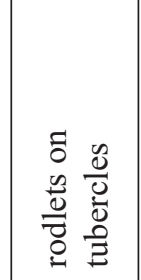 & 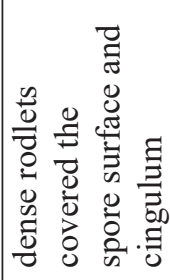 & 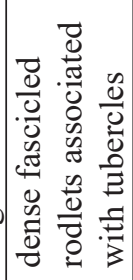 & 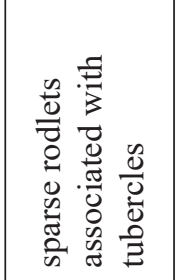 & 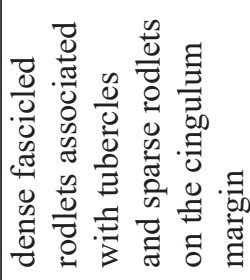 \\
\hline 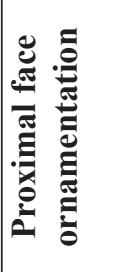 & 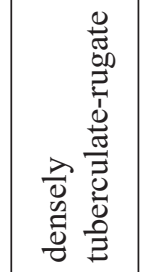 & 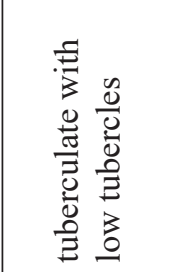 & 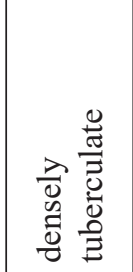 & 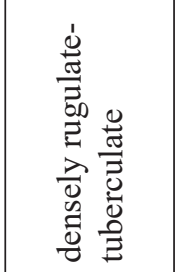 & 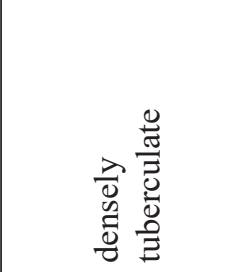 \\
\hline 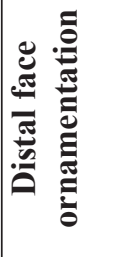 & 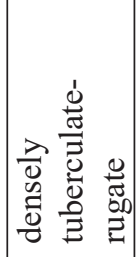 & 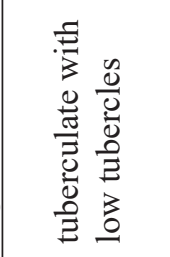 & 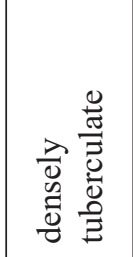 & 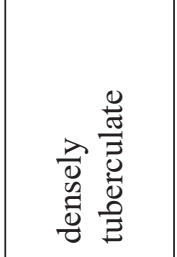 & 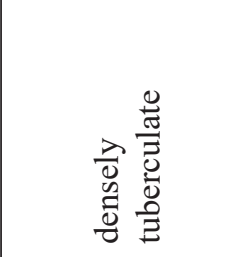 \\
\hline 葋 & 1 & 1 & & 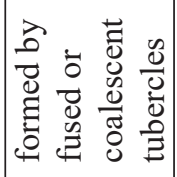 & 1 \\
\hline 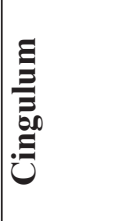 & 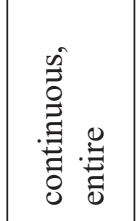 & 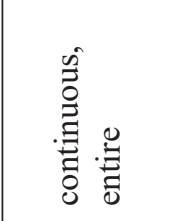 & 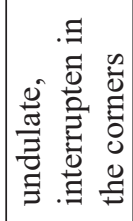 & 1 & 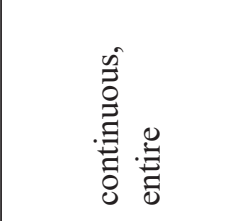 \\
\hline 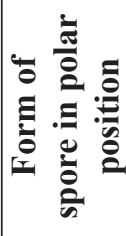 & 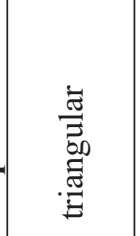 & 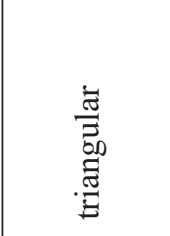 & 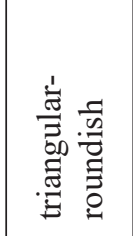 & 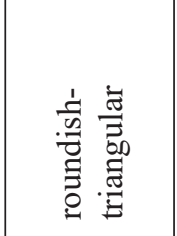 & 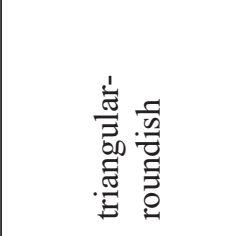 \\
\hline 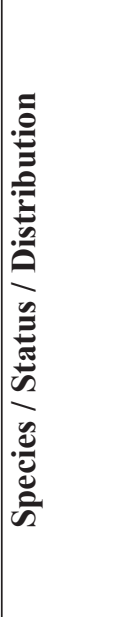 & 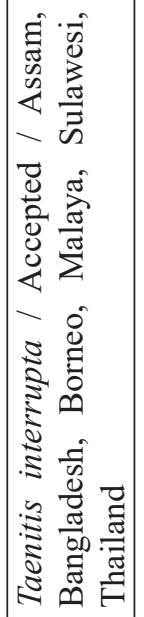 & 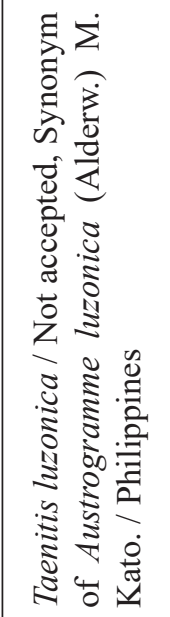 & 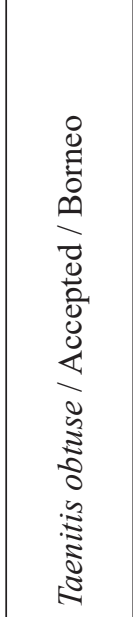 & 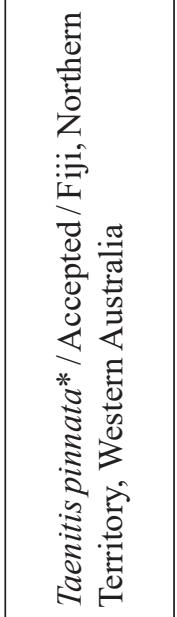 & 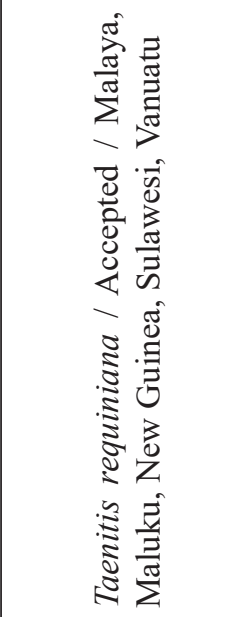 \\
\hline
\end{tabular}


Table 2

Size of spores and their elements in Austrogramme, Syngramma, and Taenitis species, mean (min-max)

\begin{tabular}{|c|c|c|c|c|c|}
\hline Species & $\begin{array}{c}\text { Equatorial } \\
\text { diameter, } \mu \mathrm{m}\end{array}$ & $\begin{array}{c}\text { Polar axis, } \\
\mu \mathrm{m}\end{array}$ & $\begin{array}{c}\text { Laesura length, } \\
\mu \mathrm{m}\end{array}$ & $\begin{array}{c}\text { Laesura } \\
\text { width, } \mu \mathrm{m}\end{array}$ & $\begin{array}{l}\text { Cingulum } \\
\text { width, } \mu \mathrm{m}\end{array}$ \\
\hline $\begin{array}{l}\text { Austrogramme } \\
\text { boerlageana* }\end{array}$ & $26.0(22.5-29.4)$ & ND & $13.2(11.8-14.4)$ & $0.8(0.6-1.1)$ & - \\
\hline $\begin{array}{l}\text { Austrogramme } \\
\text { decipiens }\end{array}$ & $27.7(22.8-31.2)$ & ND & $12.8(12.1-13.6)$ & $0.7(0.6-0.9)$ & - \\
\hline $\begin{array}{l}\text { Austrogramme } \\
\text { francii }\end{array}$ & $24.8(23.0-36.7)$ & $15.8(14.8-16.8)$ & $9.6(8.9-10.5)$ & $0.7(0.6-0.9)$ & - \\
\hline $\begin{array}{l}\text { Austrogramme } \\
\text { marginata }\end{array}$ & $24.8(23.4-25.9)$ & $19.2(18.5-20.0)$ & $11.5(10.7-12.4)$ & $0.4(0.3-0.6)$ & - \\
\hline $\begin{array}{l}\text { Syngramma } \\
\text { alismifolia* }\end{array}$ & $31.9(29.8-33.4)$ & $24.8(24.2-25.3)$ & $7.6(6.9-8.3)$ & $0.7(0.4-0.9)$ & - \\
\hline $\begin{array}{l}\text { Syngramma } \\
\text { borneensis }\end{array}$ & $30.8(28.0-33.0)$ & $28.0(27.6-28.3)$ & $11.7(10.8-11.4)$ & $1.13(1.0-1.3)$ & - \\
\hline $\begin{array}{l}\text { Syngramma } \\
\text { cartilagidens }\end{array}$ & $30.9(30.3-32.3)$ & $28.4(26.5-30.1)$ & $15.6(14.6-16.7)$ & $0.8(0.3-1.1)$ & - \\
\hline Syngramma grandis & $27.3(26.0-30.5)$ & $24.9(23.6-26.2)$ & $10.8(9.3-12.5)$ & $0.7(0.6-0.8)$ & - \\
\hline $\begin{array}{l}\text { Syngramma } \\
\text { quinata* }^{*}\end{array}$ & $28.2(25.4-29.8)$ & $23.4(21.2-25.5)$ & $8.7(7.5-10.2)$ & $0.4(0.2-0.7)$ & - \\
\hline $\begin{array}{l}\text { Syngramma } \\
\text { lobbiana* }\end{array}$ & $33.9(31.2-35.5)$ & $24.3(23.3-25.0)$ & $10.5(8.6-12.6)$ & $0.7(0.4-1.0)$ & - \\
\hline $\begin{array}{l}\text { Syngramma } \\
\text { spathulata }\end{array}$ & $37.3(34.2-40.8)$ & $27.1(26.4-27.8)$ & $18.2(16.6-21.7)$ & $2.3(1.9-2.6)$ & - \\
\hline $\begin{array}{l}\text { Syngramma } \\
\text { wallichii }\end{array}$ & $31.5(30.3-33.8)$ & $28.3(27.6-29.0)$ & $17.7(16.5-19.2)$ & $1.0(0.5-1.4)$ & - \\
\hline $\begin{array}{l}\text { Taenitis } \\
\text { blechnoides* }\end{array}$ & $40.4(39.0-42.7)$ & $26.2(25.5-27.7)$ & $14.7(13.8-16.0)$ & $1.0(0.8-1.1)$ & ND \\
\hline Taenitis cordata & $51.3(49.5-54.8)$ & $40.3(38.5-42.2)$ & $17.6(16.4-18.5)$ & $1.2(1.0-1.4)$ & $\begin{array}{c}7 . .8(6.5- \\
9.2)\end{array}$ \\
\hline Taenitis diversifolia & $41.5(38.5-44.9)$ & $33.2(31.9-34.6)$ & $15.8(14.6-17.0)$ & $1.0(0.7-1.2)$ & $2.9(1.8-3.7)$ \\
\hline Taenitis hookeri* $^{*}$ & $31.0(30.2-32.4)$ & $24.4(23.5-25.6)$ & $3.1(2.1-4.6)$ & $0.6(0.5-0.7)$ & - \\
\hline Taenitis interrupta & $44.6(41.8-47.9)$ & $33.4(32.5-34.2)$ & $16.7(14.4-21.4)$ & $0.8(0.6-1.0)$ & $2.9(2.0-3.7)$ \\
\hline Taenitis luzonica & $40.9(37.1-45.4)$ & $24.4(22.2-26.5)$ & $19.9(16.4-21.8)$ & $1.6(1.5-1.9)$ & $8.8(8.0-9.4)$ \\
\hline Taenitis obtusa & $41.9(37.0-46.3)$ & $31.7(29.4-33.6)$ & $13.9(12.2-15.1)$ & $0.9(0.5-1.2)$ & $3.1(2.3-4.0)$ \\
\hline Taenitis pinnata* & $26.5(24.0-29.0)$ & $28.4(27.5-29.2)$ & $10.3(10.1-10.5)$ & $0.9(0.8-1.0)$ & - \\
\hline Taenitis requiniana & $41.8(40.1-43.8)$ & $32.6(31.7-33.9)$ & $16.2(15.4-17.5)$ & $1.0(0.8-1.2)$ & $2.8(2.3-3.2)$ \\
\hline
\end{tabular}

Note: '-' means lack of the feature; ND - no data; an asterisk indicates the species with published data for spore size (Vaganov et al., 2018).

S. spatulata, and S. wallichii, microrugulate in S. lobbiana. Spores of $S$. grandis have microrugulate distal face and microverrucate proximal one. The spores of $S$. borneensis and $S$. cartilagidens have the most distinctive sculpture. The distal surface of spores of both species is low-tuberculate with regular tubercles 1.4-3.9 $\mu \mathrm{m}$ in diam.; in spores of $S$. cartilagidens, tubercles fused in three ridges situated parallel spore sides. The proximal surface of spores of $S$. cartilagidens is tuberculate with irregular tubercles 1.4-2.3 $\mu \mathrm{m}$ in diam.; proximal side of $S$. borneensis is smooth. Spores of only one species - S. quinata - have laesura arms immersed in the narrow laesural ridges that are reached to the spore corners. Surface of spores of S. lobbiana, S. spatulata, and S. wallichii are covered by scattered rodlets. Laesura arms are straight, reaching the spore corners, longest in S. spathulata and S. wallichii and shortest in S. alismifolia and S. quinata (Fig. 2-3; Table 1). The equatorial diameter of Syngramma spores ranges between $27 \mu \mathrm{m}$ to $40.8 \mu \mathrm{m}$, polar axis - between $21 \mu \mathrm{m}$ to $30 \mu \mathrm{m}$. The smallest spores are characteristic to $S$. grandis and $S$. quinata, the largest - to S. lobbiana and S. spathulata (Table 2).

Spores of Taenitis species in equatorial view have more or less flattened or depressed (in 
T. cordata) proximal part and mostly hemispherical distal part; spores of $T$. hookeri and $T$. pinnata are nearly globose. Three of the most noticeable features are characteristic for the Taenitis spores: cingulum, laesural ridges and tuberculate surface (Fig. 4-5). Seven of nine studied species have spores with cingulum: T. blechnoides, T. cordata, T. diversifolia, T. interrupta, T. luzonica, T. obtusa, and $T$. requiniana. Cingulum in spores of most species is continuous, variable in width, sometimes narrower at the corners, undulate (T. obtusa), or entire (remaining species). The widest cingulum is characteristic for T. luzonica $(8.0-9.4 \mu \mathrm{m})$, cingulum in $T$. cordata spores is wide $(6.5-9.2 \mu \mathrm{m})$ and thick (in equatorial view) (Fig. 4-5, Table 2). Spores of T. hookeri and T. pinnata are non-cingulate. Spores of three species have the prominent laesural ridges, entire in T. cordata and interrupted, formed by
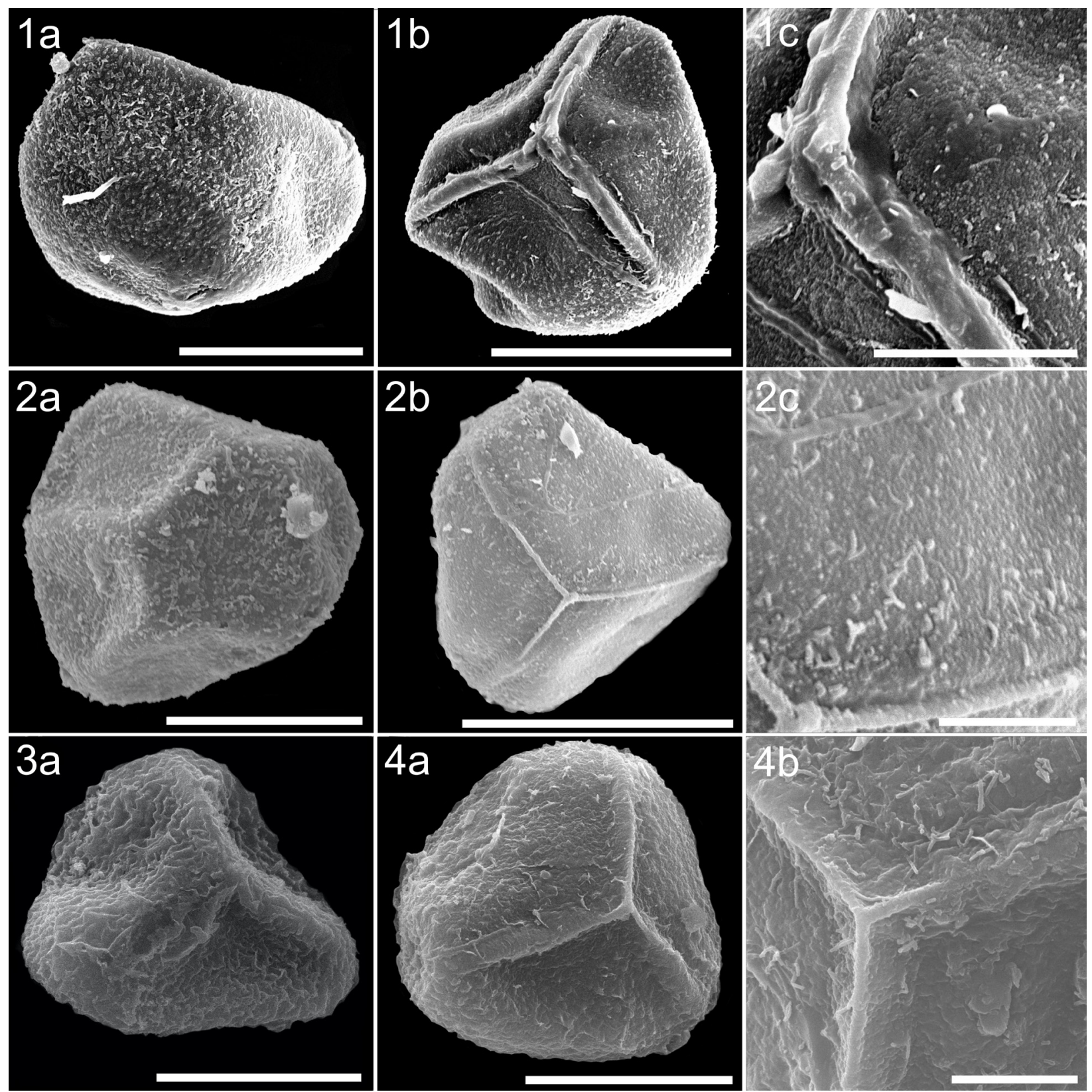

Fig. 2. SEM-micrographs of the spores of Syngramma J. Sm.: 1 - Syngramma spathulata (C. Chr.) Holttum: a - spore in equatorial position; $\mathrm{b}$ - spore in proximal-equatorial position; $\mathrm{c}$ - fragment of proximal side of spore and laesura arms; 2 - Syngramma wallichii (Hook.) Bedd: a - distal side; b - proximal side; c - fragment of proximal side of spore and laesura arms; 3 - Syngramma alismifolia (C. Presl) J. Sm.: a - spore in proximal-equatorial position; 4 Syngramma lobbiana (Hook.) J. Sm.: a - spore in proximal-equatorial position; $b$ - fragment of proximal side of spore and laesura arms. 1b, $2 \mathrm{~b}-30 \mu \mathrm{m} ; 1 \mathrm{a}, 2 \mathrm{a}, 3 \mathrm{a}, 4 \mathrm{a}-20 \mu \mathrm{m} ; 1 \mathrm{c}-10 \mu \mathrm{m} ; 2 \mathrm{c}, 4 \mathrm{~b}-5 \mu \mathrm{m}$. 

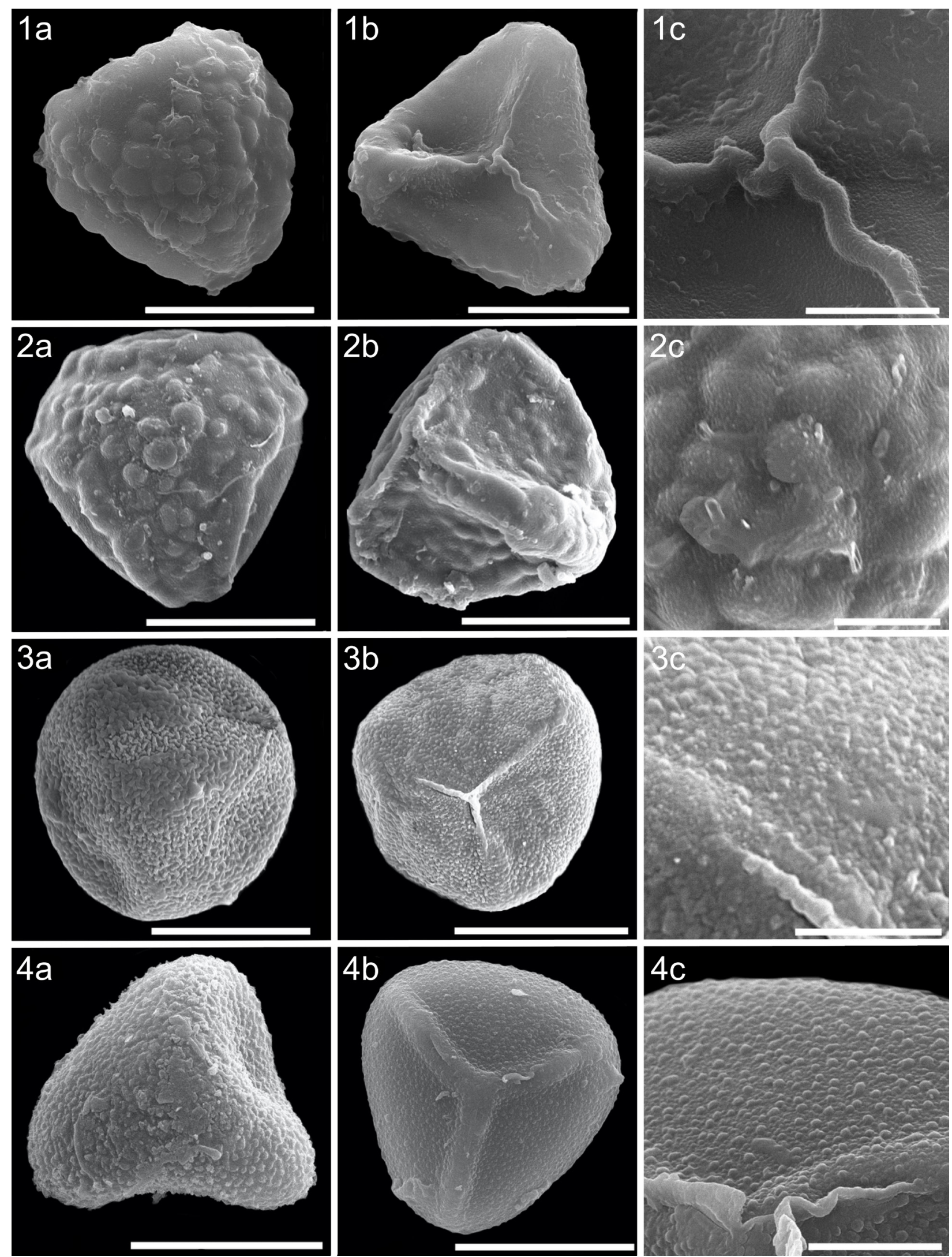

Fig. 3. SEM-micrographs of the spores of Syngramma J. Sm.: 1 - Syngramma borneensis (Hook.) J. Sm.: a - distal side; b - proximal side; c - fragment of proximal side of spore and laesura arms; 2 - Syngramma cartilagidens (Baker) Diels: $\mathrm{a}$ - distal side; $\mathrm{b}$ - spore in proximal-equatorial position; $\mathrm{c}$ - fragment of proximal side of spore and laesura arms; 3 - Syngramma grandis (Copel.) C. Chr.: a - distal side; b - proximal side; c - fragment of proximal side of spore and laesura arms; 4 - Syngramma quinata (Hook.) Carruth.: a - distal side; b - proximal side; $\mathrm{c}$ - fragment of proximal side of the spore and laesura arms. 1a, 1b, 2a, 2b, 3a, 3b, 4a, 4b-20 $\mu \mathrm{m} ; 1 \mathrm{c}, 2 \mathrm{c}, 3 \mathrm{c}, 4 \mathrm{c}-5 \mu \mathrm{m}$. 
arranged in the rows fused tubercles in spores of T. hookeri and T. pinnata. Ornamentation of distal and proximal surfaces in spores of most species is densely tuberculate, in spores of $T$. hookeri baculate, in spores of T. interrupta and T. pinnatatuberculate-rugate, and in T. cordata - rugate (Fig. 4-5). The most conspicuous character of the ornamentation of spore surfaces is the presence of rodlets associated with tubercles: rodlets are mostly fused in tufts and situated on the top of tubercles.
The most visible and dense rodlets are characteristic for spores of Taenitis diversifolia, T. luzonica, T. obtusa, T. requiniana; in $T$. diversifolia, rodlets fused in tufts and associated with the top of tubercles form especially expressed regular net (Fig. 5). The low-tuberculate surface in T. luzonica spores is densely covered mostly by adpressed rodlets. Cingulum may be smooth (T. blechnoides, T. cordata, T. interrupta) or covered by dense rodlets (T. luzonica); in $T$. diversifolia and
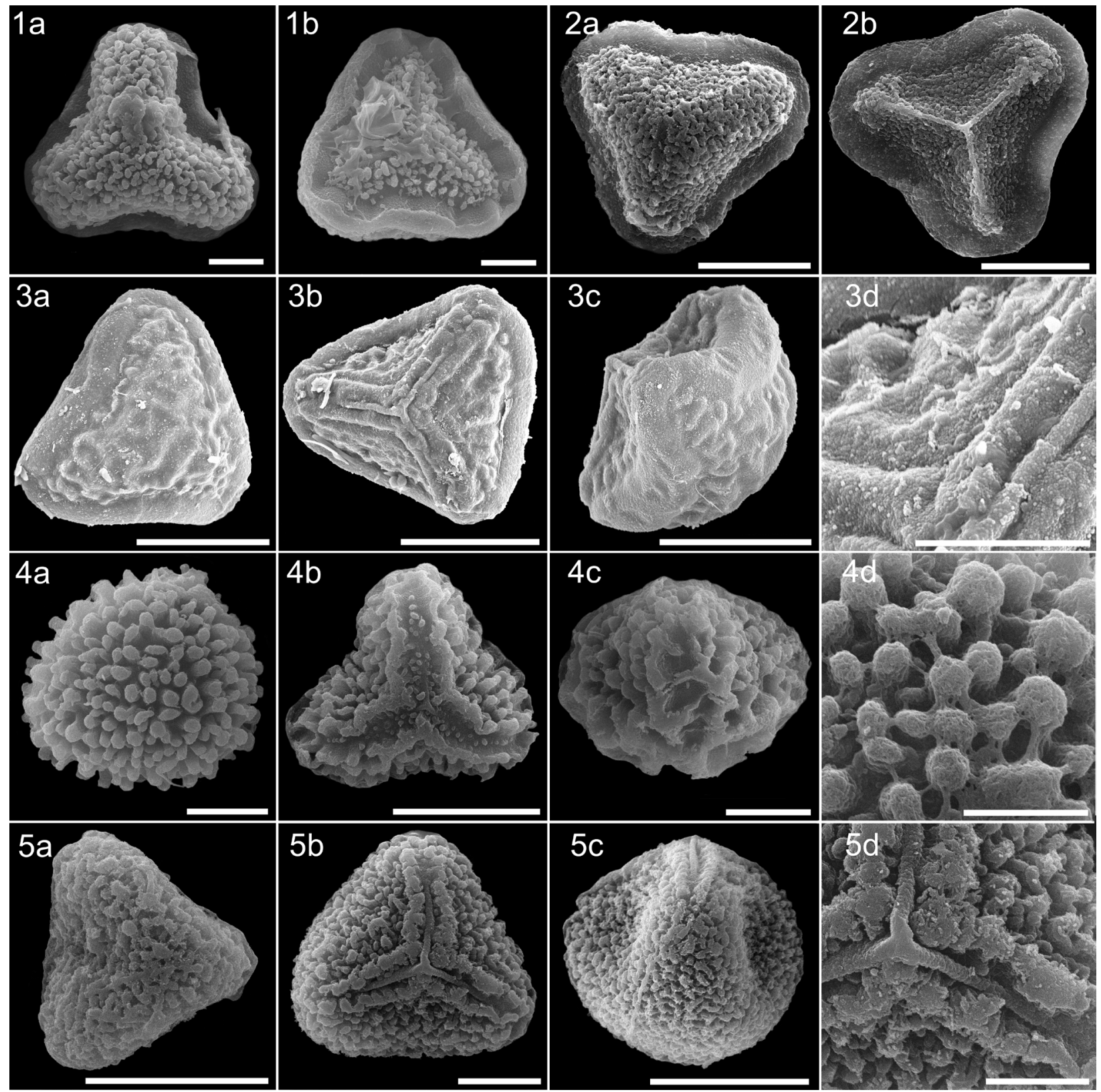

Fig. 4. SEM-micrographs of the spores of Taenitis Willd. ex Schkuhr: 1 - Taenitis blechnoides (Willd.) Sw.: a - distal side; $\mathrm{b}$ - proximal side; 2 - Taenitis interrupta Hook. et Grev.: a - distal side; b - proximal side; 3 - Taenitis cordata (Gaudich.) Holttum: a - distal side; b - proximal side; c - spore in equatorial-proximal position; $\mathrm{d}$ - fragment of equatorial-proximal side of spore and laesura arms; 4 - Taenitis hookeri (C. Chr.) Holttum: a - distal side; b - proximal side; $\mathrm{c}$ - spore in equatorial position; $\mathrm{d}$ - fragment of distal side of spore and laesura arms; 5 - Taenitis pinnata (J. Sm.) Holttum: a - distal side; $b$ - proximal side; $\mathrm{c}$ - spore in equatorial position; $\mathrm{d}$ - fragment of proximal side of spore and laesura arms. 1a, 1b, 3d, 4a, 4c, 5b - $10 \mu \mathrm{m} ; 2 \mathrm{a}, 2 \mathrm{~b}, 4 \mathrm{~b}, 5 \mathrm{a}, 5 \mathrm{c}-20 \mu \mathrm{m} ; 3 \mathrm{a}, 3 \mathrm{~b}, 3 \mathrm{c}-30 \mu \mathrm{m} ; 4 \mathrm{~d}, 5 \mathrm{~d}-5 \mu \mathrm{m}$. 
T. requiniana, rodlets situated also on the cingulum margin. Laesura arms are prominent in spores of T. interrupta and T. luzonica and are depressed and obscured by dense rodlets in spores of T. blechnoides, T. diversifolia, T. obtusa, and T. requiniana (Fig. 4-5). Spores of Taenitis species are larger than spores of Austrogramme and Syngramma, equatorial diameter ranges in most species between 40 and $51 \mu \mathrm{m}$ on average, polar axis - from 24 to $40 \mu \mathrm{m}$. Two species - Taenitis hookeri and T. pinnata - have small spores, 26-31 × 26-28 $\mu \mathrm{m}$ on average (Table 2).

On the whole, our examination of four species of Austrogramme, seven species of Syngramma, and nine species of Taenitis showed that Syngramma and Austrogramme are very similar to each other in the spore shape and ornamentation and very different in these spore characteristics of Taenitis species. Syngramma and Austrogramme have simple spores with a weakly expressed ornamentation (microverrucate or microrugulate sculpture). Only two species of Syngramma have spores with lowtuberculate ornamentation. In comparison with spores of Syngramma and Austrogramme, spores of Taenitis species have a more complex structure and noticeable ornamentation: the presence of cingulum, laesural ridges and a very clear tuberculate (as an option - baculate) or rugate sculpture.

Other authors who studied the spores of Austrogramme, Symgramma, and Taenitis indicated the same characters for the same and other species of these genera. E. Hennipman (1975) emphasized that spores of Taenitis are very different from those in Austrogramme and Syngramma, and are of two kinds either with or without a cingulum. A. Tryon and B. Lugardon (1991) described the similar features for spores of these genera. Furthermore, E. Hennipman (1975) considered that differences in chromosome number in Taenitis are associated with the presence or absence of a cingulum. On the basis of the spore morphology and chromosome number, E. Hennipman (1975) considered that Syngramma is distinguished from Taenitis and related with Austrogramme; A. Tryon et. B. Lugardon (1991) supported this disposition. Published data indicate a comparatively larger spore size than the ones we found. E. Hennipman (1975) indicated larger spore size for all studied genera: 45-70 $\mu \mathrm{m}$ for Taenitis, 40-50 $\mu \mathrm{m}$ for Syngramma, and 35-40 $\mu \mathrm{m}$ for Austrogramme. A. Tryon and B. Lugardon (1991) reported the spore size for the Taenitis and Syngramma species as 33-53 $\mu \mathrm{m}$, for Austrogram$m e-30-40 \mu \mathrm{m}$. Spores of species not involved in our studies have features specific for the species of the corresponding genera. Judging by the published photos, Austrogramme asplenioides (Holttum) Hennipman has simple spores with microverrucate surface and granular deposits on it (Hennipman, 1975), spores of T. hosei (Baker) Holttum lack a cingulum. Based on the properties of the spores and chromosome numbers, E. Hennipman (1975) indicated the relationship between Austrogramme and Syngramma; the relationship between Austrogramme and Taenitis seems less obvious, also in view of the variation of the paraphyses in the three genera studied. Based on leaf morphology, M. Kato (1993) considered Taenitis pinnata as the most primitive; the other species represent different lines of reduction and specialization.

According to database "POWO: Plants of the world online" (POWO. URL: http://powo.science. kew.org/), all studied species except Taenitis luzonica are now accepted, disagreements exist only in relation to Taenitis luzonica: the name T. luzonica is considered as a synonym of the name Austrogramme luzonica (Alderw.) M. Kato. This species was described as Syngramma luzonica Alderw. (1920). R. E. Holttum (1975) transferred it from Syngramma to Taenitis (T. luzonica), and M. Kato (1988) considered it as belonging to Austrogramme (A. luzonica). On the whole, spore morphology of three studied genera is congruent with the result of molecular-phylogenetic studies (Sánchez-Baracaldo, 2004; Cochran et al., 2014; Zhang et al., 2015, 2017), which shows the close relationship between Syngramma and Austrogramme and the position of Taenitis as a sister to Syngramma and Austrogramme (together) in the collective clade (Syngramma - Austrogramme - Taenitis). Taenitis luzonica was not involved in molecularphylogenetic studies, but by the morphology of spores, it is closer to other Taenitis species than to species of Syngramma or Austrogramme. So we support the opinion of R. E. Holttum (1975) that this taxon belongs to Taenitis ( $T$. luzonica).

The spore morphology of all investigated genera is consistent with the division into sections suggested by R. E. Holttum (1975) for Syngramma and Taenitis and by E. Hennipman (1975) for Austrogramme. The Syngramma species of the section Syngramma (S. alismifolia, S. lobbiana, S. spathulata, S. wallichii, S. guinata) have spores with poorly expressed sculpture, whereas $S$. borneensis and $S$. cartilagidens belonging to the section Toxopteris (Trev.) C. Chr. have the distinct low-tuberculate sculpture. The Taenitis species belonging to the 

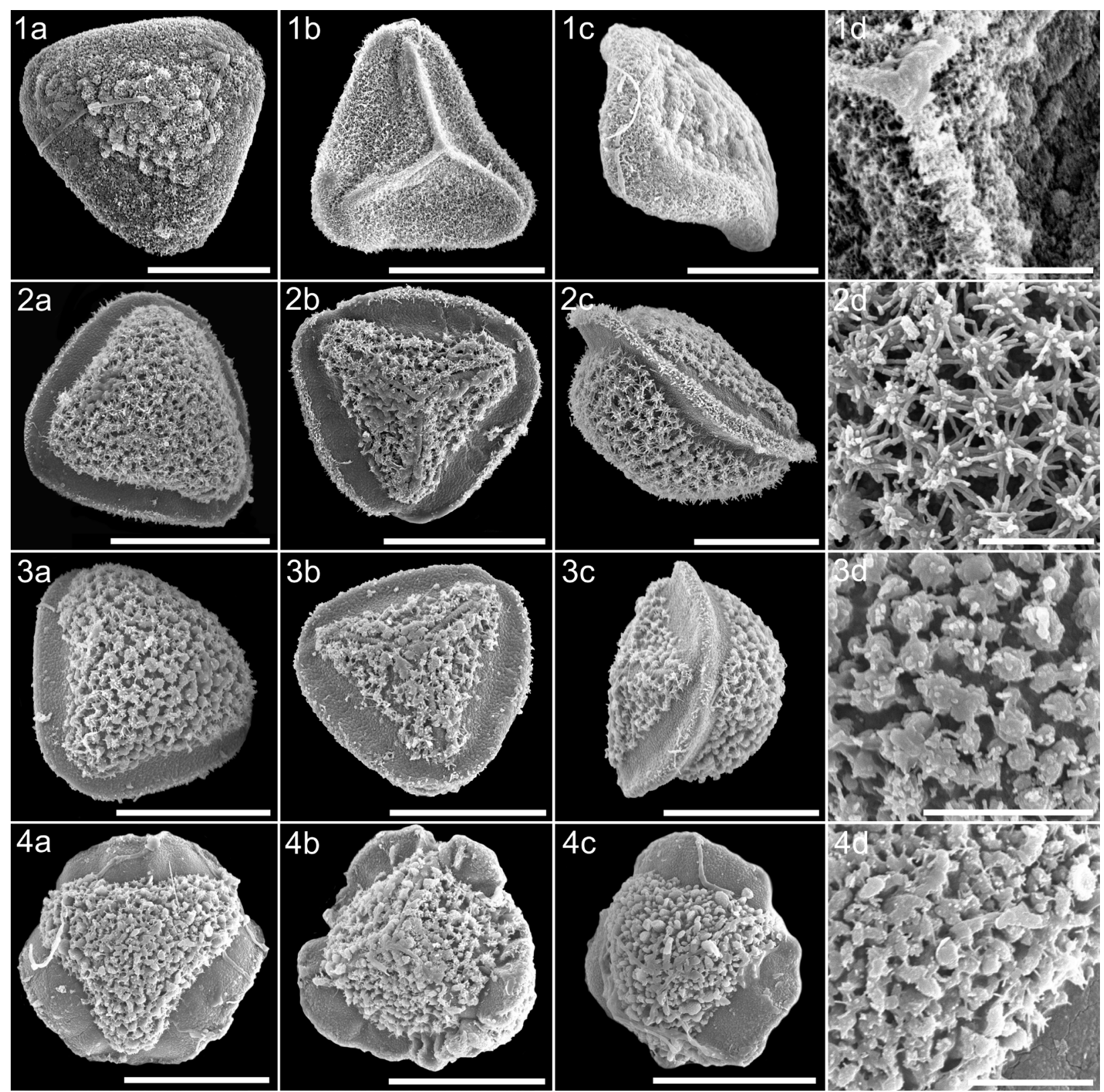

Fig. 5. SEM-micrographs of the spores of Taenitis Willd. ex Schkuhr: 1 - Taenitis luzonica (Alderw.) Holttum: a - distal side; $\mathrm{b}$ - spore in proximal-equatorial position; $\mathrm{c}$ - spore in equatorial-distal position; $\mathrm{d}$ - fragment of proximal side of spore and laesura arms; 2 - Taenitis diversifolia Holttum: a - distal side; $\mathrm{b}$ - proximal side; $\mathrm{c}$ - spore in equatorial position; $\mathrm{d}$ - fragment of distal side of spore and laesura arms; 3 - Taenitis requiniana (Gaudich.) Copel.: a - distal side; $\mathrm{b}$ - proximal side; $\mathrm{c}$ - spore in equatorial position; $\mathrm{d}$ - fragment of distal side of spore and laesura arms; 4 - Taenitis obtusa Hook.: a - distal side; $\mathrm{b}$ - proximal side; $\mathrm{c}$ - spore in distal-equatorial position; $\mathrm{d}$ - fragment of distal side of spore and laesura arms. 1a, 1c, $2 \mathrm{c}-20 \mu \mathrm{m} ; 1 \mathrm{~b}, 2 \mathrm{a}, 2 \mathrm{~b}, 3 \mathrm{a}, 3 \mathrm{~b}, 3 \mathrm{c}, 4 \mathrm{a}, 4 \mathrm{~b}, 4 \mathrm{c}-30 \mu \mathrm{m} ; 3 \mathrm{~d}, 4 \mathrm{~d}-10 \mu \mathrm{m} ; 1 \mathrm{~d}, 2 \mathrm{~d}-5 \mu \mathrm{m}$.

section Taenitis (T. blechnoides, T. interrupta, T. obtusa) have spores with cingulum close in width, without laesural ridges. Spores of T. hookeri and T. pinnata belonging to the section Loxodictyum Holttum lack cingulum but have interrupted laesural ridges formed by arranged in rows fused tubercles. The only one species - Taenitis luzonica - attributed by R. E. Holttum (1975) to the section Loxodictyum did not correspond in the spore morphology to other species of this section: its spores have relatively wide cingulum, but lack laesural ridges, the entire surface of the spore including cingulum is densely covered with rodlets. The members of the section Platytaenia (Kuhn) Holttum - T. requiniana and T. diversifolia - have very similar spores with cingulum, densely tuberculate sculpture, and dense fascicled rodlets associated with tubercles and situated on the cingulum margin as well. Taenitis 
cordata (section Schizolepton (Fée) Holttum) have spores with cingulum, entire laesural ridges, rugate sculpture, and scattered granular deposits rather than rodlets. Species of Austrogramme belonging to different sections have not so well-expressed differences in the spore morphology. Species from type section (A. fancii and A. marginata) have the smallest spores with scattered verrucae whereas species from the section Asplenopsis (Kuhn) Hennipman (A. boerlageana and A. decipiens) have spores with dense regular disposed microverrucae. As shown by E. Hennipman (1975), spores of A. asplenioides have the same sculpture.

\section{Conclusion}

The results of this study confirm the diagnostic value of the spore morphology for the phylogeny and taxonomy of three fern genera-Austrogramme, Syngramma, and Taenitis. Spores of Austrogramme and Syngramma are the simplest in ornamentation and similar to each other, whereas spores of the Taenitis species are very different from spores of Austrogramme and Syngramma. This corresponds to molecular-phylogenetic studies according to which Austrogramme and Syngramma have a closer relationship to each other, and Taenitis takes a sister position to both of them. Differences in the spore morphology correlate with the division of genera into sections.

\section{Acknowledgements}

We are grateful to Germinal Rouhan, curator of Ferns et Lycophytes of the Paris vascular plant Herbarium (P, Muséum National d'Histoire Naturelle, MNHN - Paris) for individual approach, advice on the tropical fern group and help in working with the collection, as well as curators of Herbarium of Institute of Botany of Chinese Academy of Sciences (PE, Beijing) for the possibility of obtaining the spores from the herbarium material.

The study was carried out within the framework of the State Assignments of the Ministry of Science and Higher Education of the Russian Federation: A. V. Vaganov and A. I. Shmakov (Altai State University) - project No. FZMW-2020-0003, I. I. Gureyeva (Tomsk State University) - project No. 0721-2020-0019, A. V. Vaganov (Botanical Garden-Institute of the FEB RAS) - project No. AAAA-A20-120031990009-4.

\section{REFERENCES}

Chao Y.-Sh., Huang Y.-M. 2018. Spore morphology and its systematic implication in Pteris (Pteridaceae). PLoS ONE 13(11): e0207712. DOI: 10.1371/journal.pone.0207712

Christenhusz M. J. M., Zhang X.-Ch., Schneider H. 2011. A linear sequence of extant families and genera of lycophytes and ferns. Phytotaxa 19: 7-54. DOI: 10.11646/phytotaxa.19.1.2

Cochran A. T., Prado J., Schuettpelz E. 2014. Tryonia, a new taenitidoid fern genus segregated from Jamesonia and Eriosorus (Pteridaceae). PhytoKeys 35: 23-43. DOI: 10.3897/phytokeys.35.6886

Gureyeva I. I., Kuznetsov A. A. 2015. Spore morphology of the north Asian members of Cystopteridaceae. Grana 54(3): 213-235. DOI: 10.1080/00173134.2015.1048824

Hennipman E. 1975. A redefinition of the gymnogrammoid genus Austrogramme Fournier. Fern Gaz. 11(2-3): $61-72$.

Holttum R. E. 1975. A comparative account of the fern-genera Syngramma J. Sm. and Taenitis Willd., with discussion of their relationships to each other and to other genera. Kew Bulletin 30(2): 327-343.

IPNI [2021]. International Plant Names Index. Kew: The Royal Botanic Gardens; Harvard University Herbaria; Libraries and Australian National Botanic Gardens. URL: http://www.ipni.org (Accessed 08 September 2021).

Irfan M., Jan G., Jan F. G., Murad W. 2021. Taxonomy and spore morphology of selected taxa of Cheilanthoideae and Pteridoideae (Pteridaceae) from Pakistan. Microscopy Research and Technique, 1-16. DOI: 10.1002/jemt.23845

Kato M. 1988. Taenitis and allied genera of Ambon and Seram (Moluccas) and notes on taxonomic and phytogeographic relationships of Taenitis. J. Fac. Sci. Univ. Tokyo 3(14): 161-182.

Kato M. 1993. Biogeography of ferns: dispersal and vicariance. Journal of Biogeography 20(3): 265-274.

Kuznetzov A. A., Vaganov A. V., Skapcov M. V., Erst A. S. 2014. A comparative study of spore morphology of some Pteridoideae subfamily genera. Biosciences Biotechnology Research Asia 11: 17-25. DOI: 10.13005/bbra/1435

Lellinger D. B. 2002. A modern multilingual glossary for taxonomic pteridology. Pteridologia 3: 263.

Nayar B. K., Devi S. 1966. Spore morphology of the Pteridaceae. I. The Pteridoid ferns. Grana Palinologica 6: 476-479. DOI: 10.1080/00173136609430036

Palacios-Rios M., Prada C., Y Galán J. M. G., Noa J. 2017. Spore types in Mexican and Mesoamerican species of Pteris L. (Pteridaceae). Grana 56(4): 241-256. DOI: 10.1080/00173134.2016.1217038 
Pici-Sermolli R. E. G. 1977. Tentamen Pteridophytorum genera in taxonomicum ordinem redigendi. Webbia: Journal of Plant Taxonomy and Geography 31(2): 313-512. DOI: 10.1080/00837792.1977.10670077

POWO [2021]. Plants of the World Online. URL: http://www.plantsoftheworldonline.org (Accessed 26 May 2021).

PPG I. 2016. The Pteridophyte Phylogeny Group. A community-derived classification for extant lycophytes and ferns. Journal of Systematics and Evolution 54(6): 563-603. DOI: 10.1111/jse.12229

Sánchez-Baracaldo P. 2004. Phylogenetic relationships of the subfamily Taenitidoideae, Pteridaceae. American Fern Journal 94(3): 126-142. DOI: 10.1640/0002-8444(2004)094[0126:PROTST]2.0.CO;2

Schuettpelz E., Schneider H., Huiet L., Windham M. D., Pryer K. M. 2007. A molecular phylogeny of the fern family Pteridaceae: assessing overall relationships and the affinities of previously unsampled genera. Molecular Phylogenetics and Evolution 44: 1172-1185. DOI: 10.1016/j.ympev.2007.04.011

Smith A. R., Pryer K. M., Schuettpelz E., Korall P., Schneider H., Wolf P. G. 2006. A classification for extant ferns. Taxon 3(55): 705-731. DOI: 10.2307/25065646

Tryon A. F., Lugardon B. 1991. The spores of pteridophytes: surface, wall structure, and diversity based on electron microscopy studies. Berlin: Springer. 648 pp.

Tryon R. M. 1986. Some new names and combinations in Pteridaceae. American Fern Journal 76(4): 184-186.

Tryon R. M., Tryon A. F., Kramer K. U. 1990. Pteridaceae. In: Pteridophytes and Gymnosperms. The families and genera of vascular plants. K. U. Kramer, P. S. Green (eds). Berlin: Springer Verlag. Pp. 230-256.

Vaganov A. V. 2016. A comparative study of spore morphology of the subfamily Cryptogrammoideae genera. Biological Bulletin of Bogdan Chmelnitskiy Melitopol State Pedagogical University 6(3): 333-346. DOI: $10.15421 / 2016103$

Vaganov A. V., Gureyeva I. I., Kuznetsov A. A., Shmakov A. I., Romanets R. S. 2017a. Spore morphology of the representatives of the subfamily Ceratopteridoideae (J. Sm.) R. M. Tryon from the family Pteridaceae E. D. M. Kirchn. (Pteridophyta). Ukrainian Journal of Ecology 7, 2: 124-129. DOI: 10.15421/2017_29

Vaganov A. V., Gureyeva I. I., Kuznetsov A. A., Romanets R. S. 2017b. Spore morphology of Vaginularia Fée species (Pteridaceae) from South-Eastern Asia. Ukrainian Journal of Ecology 7, 4: 231-233. DOI: 10.15421/2017_110

Vaganov A. V., Gureyeva I. I., Kuznetsov A. A., Romanets R. S. 2017c. Spore morphology of Haplopteris C. Presl species (Vittarioideae, Pteridaceae) from China. Ukrainian Journal of Ecology 7, 4: 290-294. DOI: 10.15421/2017_118

Vaganov A. V., Gureyeva I. I., Shmakov A. I., Kuznetsov A. A., Romanets R. S., König V. A. 2017d. Spore morphology of Pityrogramma calomelanos (L.) Link (Pteridaceae). Turczaninowia 20, 3: 95-102. DOI: 10.14258/ turczaninowia.20.3.9

Vaganov A. V., Gureyeva I. I., Shmakov A. I., Kuznetsov A. A., Romanets R. S. 2018a. Spore morphology of Parahemionitis arifolia (Cheilanthoideae, Pteridaceae). Turczaninowia 21, 3: 72-76. DOI: 10.14258/turczaninowia.21.3.9

Vaganov A. V., Gureyeva I. I., Shmakov A. I., Kuznetsov A. A., Romanets R. S. 2018b. Spore morphology of Taenitis, Syngramma and Austrogramme species (Pteridoideae, Pteridaceae) from South-Eastern Asia. Turczaninowia 21, 3: 5-11. DOI: 10.14258/turczaninowia.21.3.1

Zhang L., Rothfels C. J., Ebihara A., Schuettpelz E., Pechon T. Le, Kamau P., He H., Zhou Xin-Mao, Prado J., Field A., Yatskievych G., Gao Xin-Fen, Zhang Li-Bing. 2015. A global plastid phylogeny of the brake fern genus Pteris (Pteridaceae) and related genera in the Pteridoideae. Cladistics 31: 406-423. DOI: 10.1111/cla.12094

Zhang L., Zhou X.-M., Thi Lu N., Zhang L.-B. 2017. Phylogeny of the fern subfamily Pteridoideae (Pteridaceae; Pteridophyta), with the description of a new genus: Gastoniella. Molecular Phylogenetics and Evolution 109: 59-72. DOI: 10.1016/j.ympev.2016.12.037

Specimens investigated

Austrogramme boerlageana (Alderw.) Hennipman: Ambon 1939 (PE, № 01715225).

Austrogramme decipiens (Mett.) Hennipman: Sector OCE (Oceania). № 1608. Mont Humbold (Nouvelle Caledonie). 12 octobre 1869 (P, P01577226).

Austrogramme francii (Rosenst.) Hennipman: Sector OCE (Oceania). New Caledonia. Forêt de Tao. Coll.: Franc. № 1427. 1910-1. Alt. 500 (P, TYPE, P00607896).

Austrogramme marginata E. Fourn.: Sector OCE (Oceania). New Caledonia. Bords des ruisseaux à Balade. Coll.:

E. Vieillard. № 1642. 1855/1860 (P, ISOTYPE, P00607898).

Syngramma alismifolia (C. Presl) J. Sm.: Borneo E., 1953 (PE, № 01715222).

Syngramma borneensis (Hook.) J. Sm. Malaysia, Sarawak. № 56 (PE, № 01715227).

Syngramma cartilagidens (Baker) Diels: Sector OCE (Oceania). Borneo. Indonesia. Banting, Sarawak, Borneo. Coll.: Christ. 1919-2-9 (P, P01319602); dt.: 1936/05/31. Tokone. Kota Tinggi. Malay Pennisula. Kota Tinggi. 31.05 .36 (PE, № 01715228).

Syngramma grandis (Copel.) C. Chr.: Sector OCE (Oceania). Solomon. Bougainville Island: Kugumaru, Buin. Coll.: S. F. Kajewski. № 1869. 1930-6-28. Alt. 150 (P, P01313719).

Syngramma quinata (Hook.) Carruth.: № 6943. V. Guinen Japen, № 01715087; Selebes, G. Kjellberg, 1939,500 
alt. (PE, № 01715234).

Syngramma lobbiana (Hook.) J. Sm.: № 933 Native collector. Plants of Sarawak, Borneo. Collected through the Museum for the Bureau of Science, Manila, P. I. (PE, № 01715231).

Syngramma spathulata (C. Chr.) Holttum: Secteur ASI (Asie). Jamindan Capiz Province Panay. № 31307. Coll.: M. Ramos, G. Edano. April-May 1918 (P, P01398822).

Syngramma wallichii (Hook.) Bedd. Secteur ASI (Asie). Cagayan Prov., Luzon. Coll.: Maximo Ramos. Mar. 1909 (P, P01398821).

Taenitis cordata (Gaudich.) Holttum: Sector ASI (Asia). Indonesia, Rawak. 1823. Coll.: C. Gaudichaud. (P, P00607823).

Taenitis blechnoides (Willd.) Sw.: Hainan. Shan Mong, Fairly common: dry, gentle slope, clay, thicket, erect. Coll. Lau S. K., Det. E. D. Merrill, Dec. 23, 1933. № 2935 (LE).

Taenitis diversifolia Holttum: Sector OCE (Oceania). №4881. Alt. 100 ft. Maramasike. A. F. Braithwaite 26.11.1965 (P, P01482652).

Taenitis hookeri (C. Chr.) Holttum: Plants of Netherlands New Guinea. 1200 m alt. Feb. 1939 (PE, № 01715229). Taenitis interrupta Hook. et Grev.: Indonesia. Coll.: A. H. G. Alston. № 13127. dt.: 1954/01/20 (PE, № 01595231). Taenitis luzonica (Alderw.) Holttum: Sector ASI (Asia). Philippines. Guinayangan, province of Tayabas, Luzon. L. Escritor. 1913-3 (P, P01308663).

Taenitis obtusa Hook.: Sector ASI (Asia) (P, P01467801).

Taenitis pinnata (J. Sm.) Holtt.: Plants of Papua (British New Guinea) Lake Daviumbu. Rain forest. August, 1936 (PE, № 01715232).

Taenitis requiniana (Gaudich.) Copel.: Sector ASI (Asia). Indonesia, Rawak. Coll.: C. Gaudichaud. (P, P00607819). 